\title{
Fluid/structure interaction analysis using the Smoothed Particle Hydrodynamic method
}

\author{
D. Delsart ${ }^{1}$, N. Toso-Pentecôte ${ }^{2}$, A. Vagnot ${ }^{3}$, L. Castelletti ${ }^{4}$, \\ U. Mercurio ${ }^{5} \& \mathrm{~S}$. Alguadich ${ }^{5}$ \\ ${ }^{I}$ Office National d'Etudes et de Recherches Aérospatiales (ONERA), \\ France \\ ${ }^{2}$ German aerospace Center (DLR), Institute of Structures and Design, \\ Germany \\ ${ }^{3}$ EUROCOPTER, Airframe Stress Department, France \\ ${ }^{4}$ Politecnico di Milano, Dipartimento di Ingegneria Aerospaziale, Italy \\ ${ }^{5}$ CIRA ScpA, Centro Italiano ricerche Aerospaziali, Italy
}

\begin{abstract}
Works presented in this paper have been performed within the GARTEUR Action Group AG15 "Improvement of SPH methods for application to helicopter ditching" whose overall objective is aimed at assessing analytical tools for the simulation of helicopter impacts on water. It particularly focused on the Smoothed Particle Hydrodynamic (SPH) formulation, which consists of a gridless Lagrangian method and whose main interest, with respect to fluid/structure interaction issues, relies upon the absence of connectivity between the "particle elements", thus permitting it to cope with large deformations without generating mesh distortion problems. In the first step, the SPH method was evaluated through the simulation of droplet impact tests onto a rigid plate, performed at two impact velocities $(1 \mathrm{~m} / \mathrm{s}$ and $5 \mathrm{~m} / \mathrm{s})$; numerical results were analysed in terms of force and impulsion data and proved to conveniently fit with the test results. In the second step, water impact tests on simple shapes were simulated, including tests on triangular and cylindrical rigid shapes, performed at the Politecnico Di Milano, and vertical drop tests of a deformable metallic cylinder performed at CIRA. For the considered structures and impact conditions, the simulations permitted one to draw a general conclusion in terms of prediction capacities regarding deformations, accelerations and pressure data.
\end{abstract}

Keywords: smooth particles hydrodynamics, water, impact. 


\section{Introduction}

In the field of aircraft ditching modelling, classical solutions for fluid/structure interaction simulation, based on Finite Element Lagrange or even ALE (Arbitrary Lagrange Euler) formulations, face well-known limitations [1] - mesh distortions leading to numerical instabilities (negative volumes) and high CPU consumption (time step decrease) that make the modelling of specific threats such as water ingress through a failed structure - almost impossible. The present works, carried out within the GARTEUR Action Group AG15, investigate a more recent formulation based on SPH (Smooth Particles Hydrodynamics) formulation that permits one to get rid of most of the numerical issues insofar the media behaviour is no longer modelled through elements deformation, but through particles inter-displacement. Different codes available among the AG15 partners are thus investigated and the present paper gives an overview of some of the achieved results. As a first step to evaluate the SPH formulation, laboratory tests consisting of droplet impacts onto a rigid plate are considered, with the measured output limiting to the force generated by the droplet on the rigid support. In the second step, the SPH method is applied to the modelling of impact tests of simple structures, including rigid and deformable shapes, for an impact velocity range relevant with crash issues. Such tests permit one to evaluate the capacity of the method to predict standard observables, such as acceleration and pressure data.

\section{Smoothed Particle Hydrodynamic method}

The Smoothed Particle Hydrodynamics method is a gridless Lagrangian method, whose corner stones are two approximations, namely the kernel approximation and the particle approximation. The estimate $\langle\mathrm{A}(\overrightarrow{\mathbf{r}})\rangle$ of $\mathrm{A}(\overrightarrow{\mathbf{r}})$ called the kernel approximation is derived from the following identity:

$$
\begin{gathered}
\langle\mathbf{A}(\overrightarrow{\mathbf{r}})\rangle=\int_{\Omega} \mathbf{A}\left(\overrightarrow{\mathbf{r}}^{\prime}\right) \delta\left(\overrightarrow{\mathbf{r}}-\overrightarrow{\mathbf{r}}^{\prime}\right) \mathbf{d} \mathbf{r}^{\prime} \\
\int_{\Omega} \delta\left(\overrightarrow{\mathbf{r}}-\overrightarrow{\mathbf{r}}^{\prime}\right) \mathbf{d} \overrightarrow{\mathbf{r}}^{\prime}=1 \text { (delta function) }
\end{gathered}
$$

where $\mathrm{A}(\overrightarrow{\mathbf{r}})$ may be any scalar vector or tensor material property, $\overrightarrow{\mathbf{r}}$ is the position vector in space and $\Omega$ the domain of integration. Eqn (1) shows that the "value at a point" of a continuous function over a continuous domain could be extracted from its integral by using a delta function as a "filter". Assuming now that the delta function is replaced by another function $\mathrm{W}(\overrightarrow{\mathbf{r}})$, which spans a certain "range" but still obeys the basic delta function property $\lim _{\mathbf{h} \rightarrow 0} \mathbf{W}\left(\overrightarrow{\mathbf{r}}-\overrightarrow{\mathbf{r}}^{\prime}, \mathbf{h}\right)=\delta\left(\overrightarrow{\mathbf{r}}-\overrightarrow{\mathbf{r}}^{\prime}\right)$, then eqn (1) yields

$$
\langle\mathbf{A}(\overrightarrow{\mathbf{r}})\rangle=\int_{\Omega} \mathbf{A}\left(\overrightarrow{\mathbf{r}}^{\prime}\right) \mathbf{W}\left(\mathbf{r}-\overrightarrow{\mathbf{r}}^{\prime}, \mathbf{h}\right) \mathbf{d} \mathbf{r}^{\prime}
$$


Eqn (3) is similar in appearance to eqn (2) except for the function W, which is called the kernel function and the range of influence it spans is controlled by the "smoothing length" $h$. It means that the value of a function at a point contains information not just about that point but also about the range around that point that the kernel in question is spanning. Therefore, a "smoothing" of the domain has taken place, hence the term "smoothed" particle hydrodynamics.

The particle approximation implies that the domain around the point in question where we seek to define the value of a function is not continuous. Instead it consists of a number of "topologically unconnected finite elements" called particles in order to distinguish them from the classical finite elements, which have a pre-defined and rigid topological connection ("connectivity" defined at the input level). The consequence of this approximation is the replacement of the integral by a summation over a finite number of particles, which are also interpolation points, and the modification of the algebra to account for the "number density" of the domain (i.e. how many particles can be found within a given domain volume defined by the kernel). This is expressed as below:

$$
\langle\mathbf{A}(\overrightarrow{\mathbf{r}})\rangle=\sum_{\mathbf{j}=1}^{\mathbf{J}}\left(\mathbf{m}_{\mathbf{j}} / \boldsymbol{\rho}_{\mathbf{j}}\right) \mathbf{A}_{\mathbf{j}} \mathbf{W}\left(\left|\overrightarrow{\mathbf{r}}-\overrightarrow{\mathbf{r}}_{\mathbf{j}}\right|, \mathbf{h}\right)
$$

where $A_{j}=A\left(\vec{r}_{j}\right), m_{j}$ and $\rho_{j}$ are respectively the mass and material density associated with the particle $\mathrm{j}, \overrightarrow{\mathbf{r}}_{\mathrm{j}}$ the position vector of particle $\mathrm{j}$ and $\mathrm{J}$ the number of particles that are within the region of influence of a given particle at a given time. Eqn (4) indicates that the contribution of each particle within the kernel range (taking into account its number density) is summed over all the particles in order to produce the smoothed value of a function at a point. In order for the kernel and particle approximations to be pragmatic, the choice of the kernel function should be such that following properties are satisfied:

- Compact form i.e. acting over a finite range, zero outside that range

- Positive within this range,

- Respecting the "delta function properties",

- Monotonically decreasing,

- Degenerating in the limit to a delta function.

One should note that the first two requirements listed above are the same for the classical interpolation functions of the FE method. Therefore, the kernel should be seen as a form of interpolation function. A more detailed discussion regarding the kernel choice, the control of the smoothing length and other numerical issues can be found in [2]. Figure 1 illustrates graphically the similarity between the FE and the SPH approximations. A patch of 9 elements is shown in both the FE and the equivalent SPH approximation. The interpolation functions have been overlaid upon the central element of the FE mesh while the kernel of the central particle has been "sketched" as spanning its neighbours in the SPH mesh.

The main advantage of the SPH method is the absence of connectivity between the "particle elements", which therefore permits to treat large 

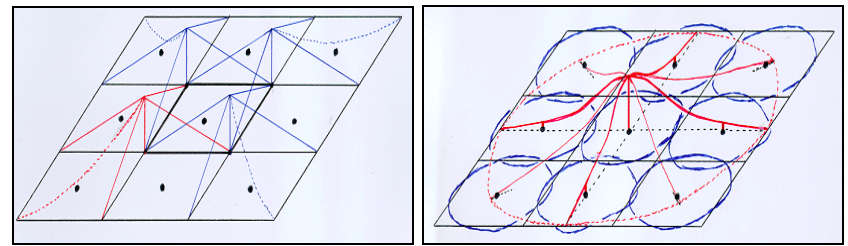

Figure 1: Comparison between a) FE and b) SPH (9 elements patch modelling).

deformation. In particular, problems of mesh distortion and tangling common with the standard Finite-Element method can be avoided. Furthermore, as both FE and SPH are Lagrangian techniques, penalty contact algorithms already available for the Finite-Element method can also be used to define contacts between SPH particles and classical Finite-Elements. However, a major drawback of this method is the very high computation time due to the necessary search for neighbours around each of the particles, which should occur periodically. Based on these facts, one strategy to simulate the impact of structures on water is to combine SPH (in the impact area) and classical finiteelement solids (around the impact area) to model the water continuum, with the transition from particles to solid elements defined as a tied interface. This strategy enables to limit the number of particles and consequently positively influences the computation time.

\section{Simulation of droplet impact tests on rigid plates}

\subsection{Droplets impact tests onto a rigid plate}

In a preliminary step to evaluate the SPH method, impact tests of a droplet onto a rigid plate have been performed. The circular aluminium plate $(\varnothing 20 \mathrm{~mm})$ was mounted on a low capacity piezo-electric load cell to record the evolution of the impact force. Water droplets were generated by a needle permitting to control the droplet diameter and could be dropped from a height up to $2 \mathrm{~m}$ thus permitting to cover an impact velocity range from 0,5 up to $5 \mathrm{~m} / \mathrm{s}$. In the present paper, two impact velocities are considered -1.05 and $5.17 \mathrm{~m} / \mathrm{s}-$ with a $\varnothing=4.2 \mathrm{~mm}$ droplet as illustrated in the following figure with the measured force versus time (pictures on the right are for the $5.17 \mathrm{~m} / \mathrm{s}$ impact velocity).

\subsection{Simulation results of the droplet impact test at $5.17 \mathrm{~m} / \mathrm{s}$}

The droplet is meshed with a hexagonal network defined with an inter-particle distance of $0.1 \times \sqrt{ } 2$ so as to obtain a smoothing length equal to 0.1 , thus leading to a model with 54694 particles. Two simulations are performed, for 2 values of the friction coefficient -0 and 0.2 - of the contact interface managing the interaction between the droplet and the plate (modelled with shell elements fully constrained in displacement and rotation) and are run over a $2 \mathrm{~ms}$ duration. The numerical force/time curves are post-treated from the contact interface normal 
force and are presented in the following figure, together with the corresponding experimental data (the animation pictures correspond to the simulation with the friction coefficient set to 0 ). Results show that the profile of the force/time signal is well predicted by the simulations though its magnitude tends to be globally overestimated. One also notes that the friction coefficient does not affect the droplet behaviour as both simulations lead to super-imposable results.

In Table 1, results are analysed in terms of maximum force and time of appearance of this maximum force, and in terms of maximum impulsion (integral of the force/time signal). This shows that the magnitude of the maximum force is in good agreement with the test data but is postponed compared to the experiment, while the impulsion is largely over-estimated, as a consequence of the global overestimation of the force signal.

\subsection{Results of the simulation of droplet impact at $1.05 \mathrm{~m} / \mathrm{s}$}

Two simulations are also conducted at $1.05 \mathrm{~m} / \mathrm{s}$, for 2 values of the friction coefficient, and are run over a $6 \mathrm{~ms}$ run time, with the same parameters as for the
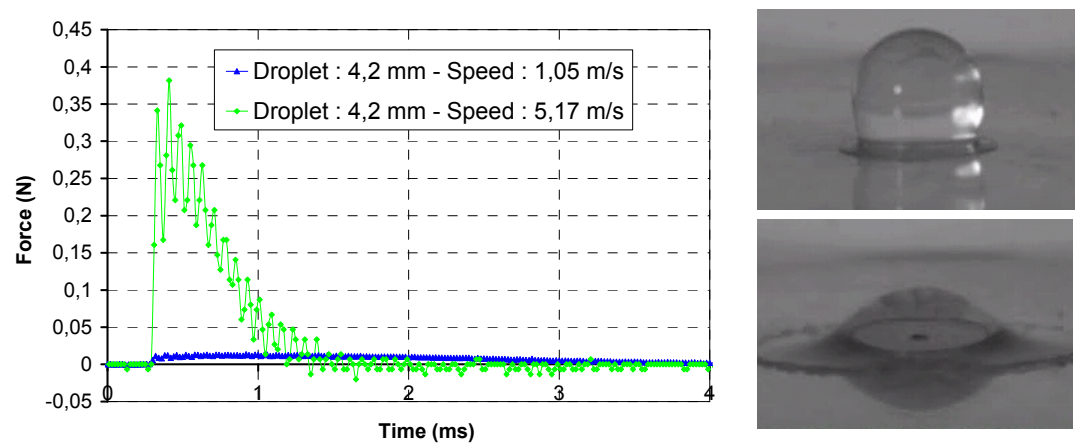

Figure 2: $\quad$ Results of the impact tests of a $\varnothing=4.2 \mathrm{~mm}$ droplet on a rigid plate.
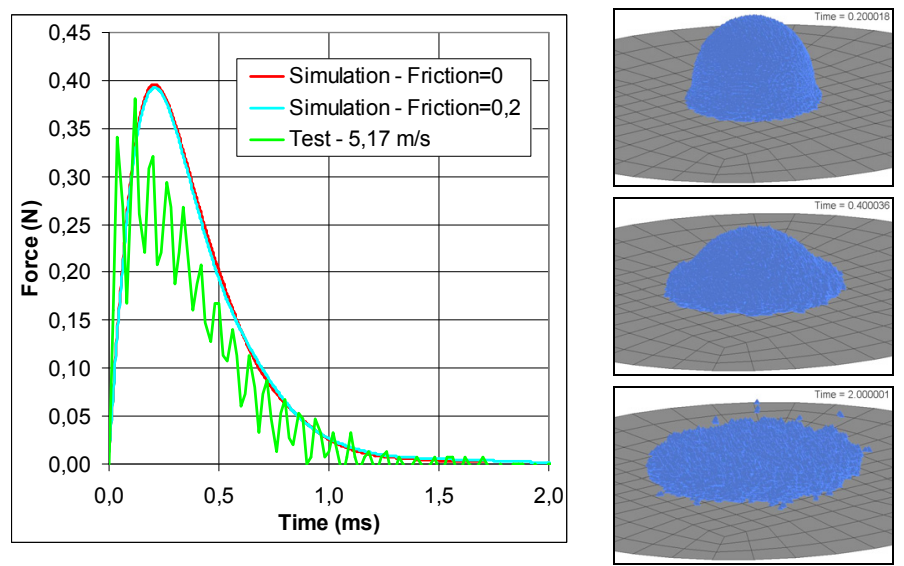

Figure 3: Comparison of the experimental/numerical results at $5.17 \mathrm{~m} / \mathrm{s}$. 
Table 1: Comparison of the experimental/numerical data at $5.17 \mathrm{~m} / \mathrm{s}$.

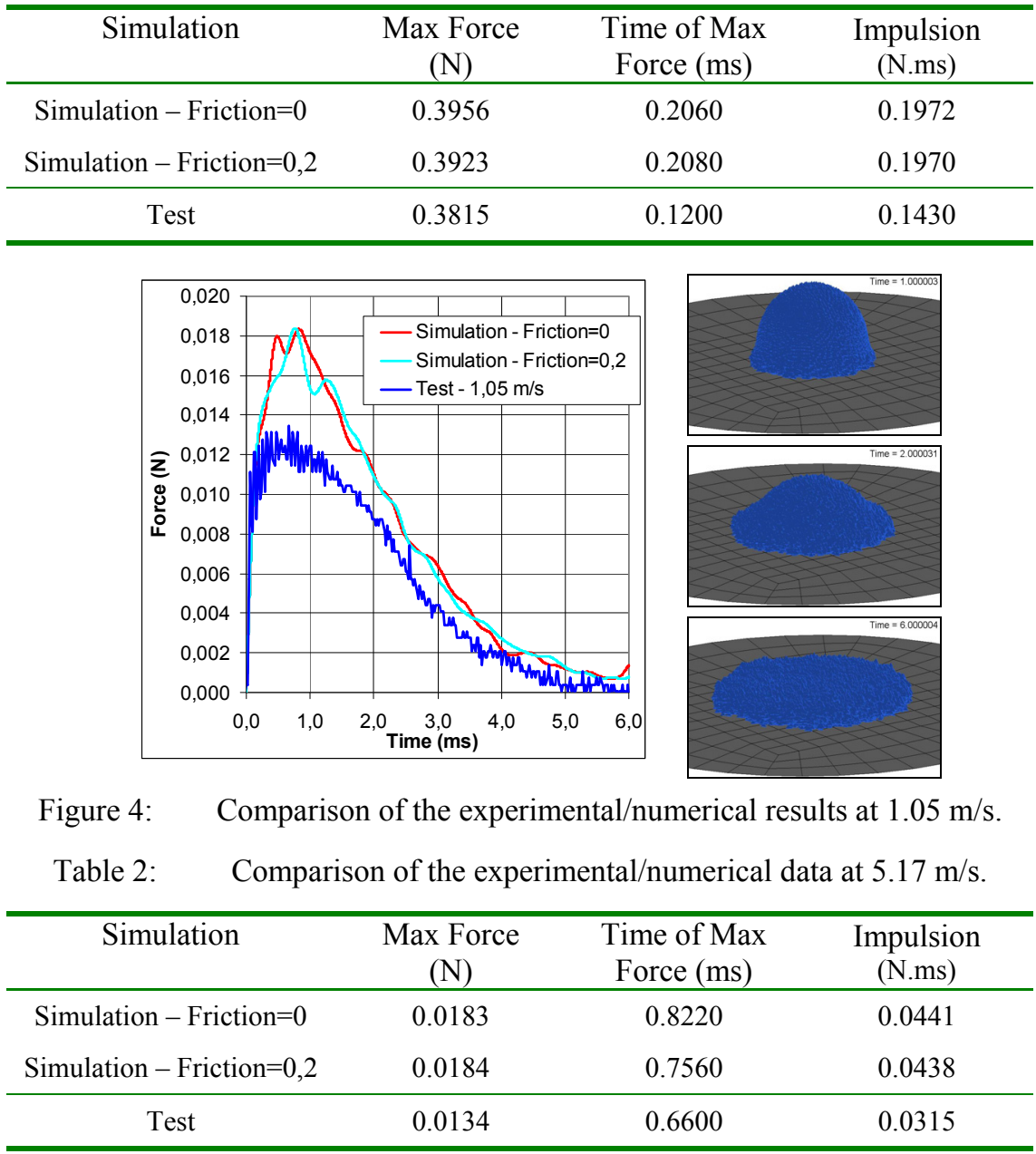

$5.17 \mathrm{~m} / \mathrm{s}$ velocity. One observes that the simulations also conveniently predict the profile of the force/time signal but again globally overestimate its magnitude.

The analysis of the maximum force and impulsion shown in the following table confirms the above conclusions, with an overestimation of the maximum force and impulsion values.

\section{Simulation of impact tests of simple structures}

\subsection{Impact tests on triangular and cylindrical rigid shapes - Politecnico Di Milano (PM)}

Politecnico Di Milano performed guided tests with triangular ( $1 \mathrm{~m}$ long and $0.4 \mathrm{~m}$ wide for a $37 \mathrm{Kg}$ weight) and cylindrical (1m long and $0.25 \mathrm{~m}$ wide for a $33 \mathrm{Kg}$ 
weight) rigid articles, in a $22 \mathrm{~m}$ height drop-tower installed upon a $8 \mathrm{~m}$ diameter pool [3]. Specimens were equipped with 3 accelerometers ( 2 at corners and 1 in the middle of one edge) in order to control the article incidence at the impact and new enhanced pressure transducers ( 8 for the triangle and 6 for the cylinder) designed and manufactured at PM - metallic cylindrical end-cups including a thin membrane equipped with a diaphragm strain gauge on its internal face (with the possibility to modify to membrane thickness to adjust the sensor sensitivity). In addition to these accelerations and pressures measurements, results also included pictures/videos.

Preliminary tests were conducted to assess the test configuration and equipments, for a large impact velocity range (up to $12.5 \mathrm{~m} / \mathrm{s}$ for the triangular shape and $7.7 \mathrm{~m} / \mathrm{s}$ for the cylindrical shape). Finally, two impact velocities were selected for the final test program $-4.4 \mathrm{~m} / \mathrm{s}$ and $7.7 \mathrm{~m} / \mathrm{s}-$ with 3 tests planned for each velocity and shape. The following figure illustrates typical results for one test on the triangular shape at the $4.4 \mathrm{~m} / \mathrm{s}$ impact velocity, noting that the pressure transducers and accelerometers provided repeatable measurements between the 3 performed tests. To evaluate the test data relevance, a comparison with analytical results obtained using Von Karman's formulas for rigid body water impacts was performed and showed good correlation. Finally, it was observed that the trend of both pressure and acceleration measurements were rather linear with the drop-height.

\subsection{Simulation results of the impact tests on triangular and cylindrical rigid shapes}

Only the simulations of the $4.4 \mathrm{~m} / \mathrm{s}$ impact test of the triangle shape and the $7.7 \mathrm{~m} / \mathrm{s}$ impact test of the cylindrical shape are presented in the following paragraph. For computation cost reasons, 1/4 of the pool is considered, with appropriate symmetry boundary conditions. The water basin is modelled by combining SPH particles in the impact area and solid Finite Elements around, modelled with an isotropic elastic plastic hydrodynamic material law. In the

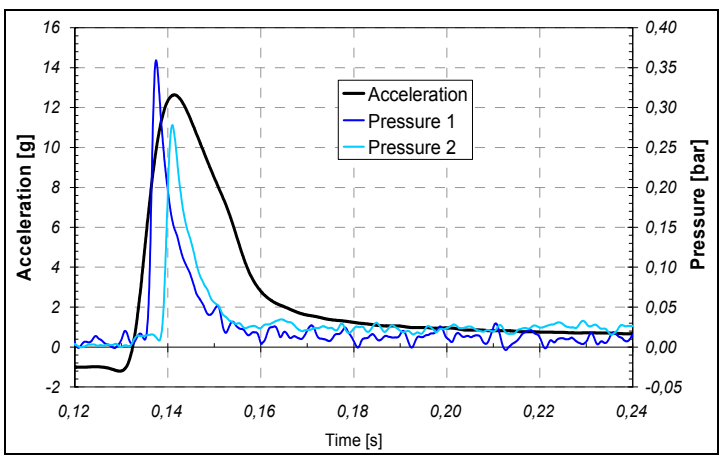

Figure 5: Impact on water of the triangular shape $-V_{\text {impact }}=4.4 \mathrm{~m} / \mathrm{s}$ (Politecnico Di Milano test facility).

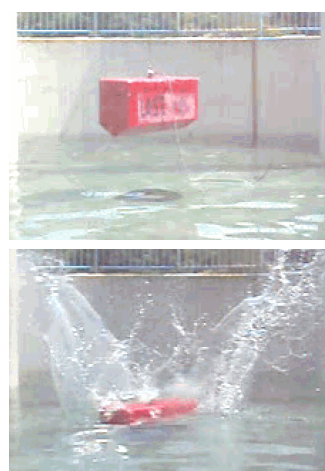


simulations, pressures are measured from the forces measured by dedicated contact interfaces managing the interaction between the particles and a set of shell elements representing the pressure sensor surface. Therefore, the SPH density was determined so as to obtain a relevant number of "particles" interacting with each pressure transducers, which led to a water model containing 150000 SPH particles and 14000 solid elements.

As for the droplet tests simulation, two values (0 and 0.2$)$ of the friction coefficient between the particles and the structure are considered.

In Figure 6, the measured acceleration time history of the wedge impacting on water at $4.4 \mathrm{~m} / \mathrm{s}$ is depicted with the simulation results. As it can be seen, variation of the friction does not show any significant influence on the acceleration in that case. The simulation predicts an acceleration peak of $14 \mathrm{~g}$ whereas the measured acceleration peak amounts to $13 \mathrm{~g}$. Acceleration peaks agree well between test and analysis. Nevertheless, it can be observed that the calculated acceleration peak is delayed compared to the measured one. Regarding the pressure time histories, measured pressure peaks are between 0.37 and 0.41 bar, whereas calculated ones are in the range $0.28-0.35$ bar; the simulation is therefore also able to predict the order of magnitude of the pressure.

Finally, Figure 6 shows an animation picture taken during the impact and especially illustrates the capacity of the SPH model to conveniently reproduce the formation of a splash around the impacting structure. Concerning the
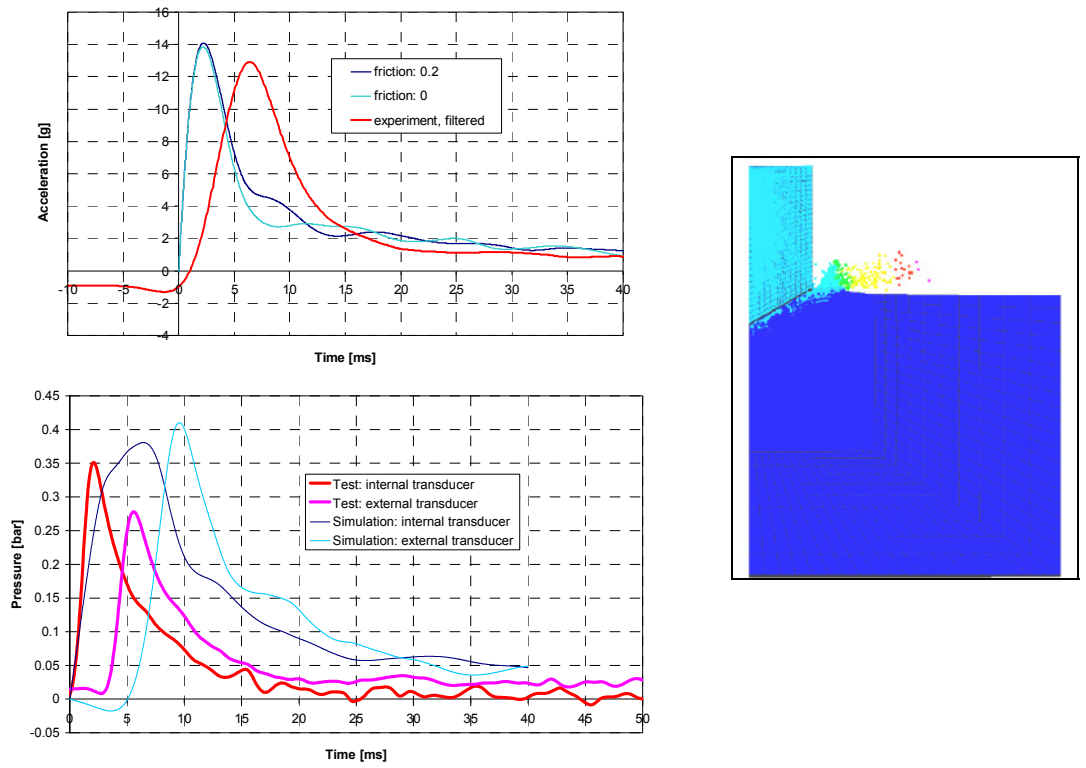

Figure 6: Comparison between test and simulation (CFC60 filter) for the 4.4 $\mathrm{m} / \mathrm{s}$ impact of a wedge. 
cylinder impacting on water, similar comparisons to the wedge case between test and simulation are performed. Figure 7 shows that the simulation is also able to predict well the acceleration peak $(37 \mathrm{~g}$ in the test -36 and $40 \mathrm{~g}$ in the simulations). Both peaks in the simulations correspond to two different frictions in the definition of the contact between the cylinder and the water continuum. Contrary to the case with the wedge, the value of the friction coefficient slightly influences the acceleration peak (17\%) - this is also observed for the impact velocity of $4.2 \mathrm{~m} / \mathrm{s}$, which is not presented in this paper. It is presumed that the cylinder due to its geometry (surface tangent at impact parallel to the water surface) presents a higher drag than the wedge when entering water and therefore leads to higher friction forces acting on the structure surface compared to the wedge. Figure 7 also shows that the order of magnitude of the pressures is satisfactorily predicted by the simulation.

Finally, the animation picture proves the capacity of the method to conveniently reproduce the formation of a splash, which is, in the cylinder case, intrinsically topologically different from the wedge case.

\subsection{Impact tests on a deformable cylindrical shape - CIRA}

CIRA conducted vertical drop tests on water of a deformable metallic structure. The tests article consisted of a half-cylinder made of $2 \mathrm{~mm}$ thick steel skin stiffened with metallic frames (resulting in a $66 \mathrm{Kg}$ weight) and was tested in the CIRA-LISA (Laboratory for Impact tests on Aerospace Structures) drop tower
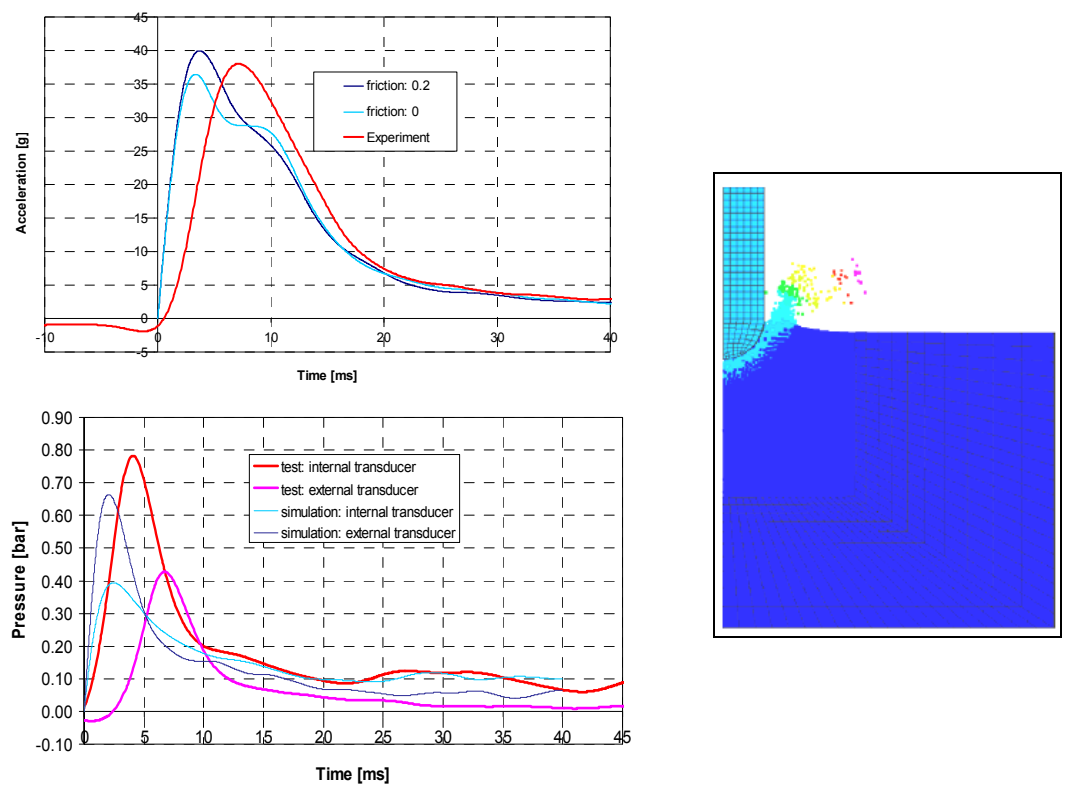

Figure 7: Comparison between test and simulation (CFC60 filter) for the 7.7 $\mathrm{m} / \mathrm{s}$ impact of a cylinder. 
facility. The tests were performed at 3 increasing velocities $(3 \mathrm{~m} / \mathrm{s}, 8 \mathrm{~m} / \mathrm{s}$ and 10 $\mathrm{m} / \mathrm{s})$, with the specimen fixed to a trolley $(174 \mathrm{Kg})$ which was guided during its free fall.

In a preliminary step, the test configurations (specimen thickness, mass and impact velocities) were identified from numerical analysis, with the objective to obtain tested specimens with or without residual deformation. Tests exhibited the targeted behaviours insofar the $2 \mathrm{~m} / \mathrm{s}$ impact velocity led to no residual deformation of the specimen skin, while the $8 \mathrm{~m} / \mathrm{s}$ and $10 \mathrm{~m} / \mathrm{s}$ permitted to obtain increasing residual deformation. Results delivered by CIRA included acceleration (4 accelerometers fixed on the upper side of the article) and pressures ( 7 transducers distributed on the external side of the skin, at stiffened and un-stiffened areas) measurements, high speed videos and the residual deformation of the article (mapping at referenced points of the deformed skin).

Finally, as a support to the numerical works, CIRA additionally conducted dynamic mechanical characterisation tests on the steel material constitutive of the skin, for a strain-rate range up to $60 \mathrm{~s}^{-1}$. This tensile tests campaign permitted to identify the reference static mechanical properties and the strain rate influence of the steel material.

\subsection{Simulation results of the impact tests on a deformable cylindrical shape}

The specimen is modelled with shell elements associated to an elastic plastic material model taking into account strain rates, with its parameters identified from the dynamic characterisation tests performed at CIRA. The water media is modelled by combining SPH particles in the impact area and solid Finite Elements around, leading to a water model containing $62100 \mathrm{SPH}$ particles and 22400 solid elements.

The comparison with the test data is performed in terms of acceleration (nodal acceleration at nodes located at the accelerometers position), pressures (simulated pressures are extracted from dedicated contact interfaces between the water media and selected shell elements on the skin) and residual deformation of the tank.
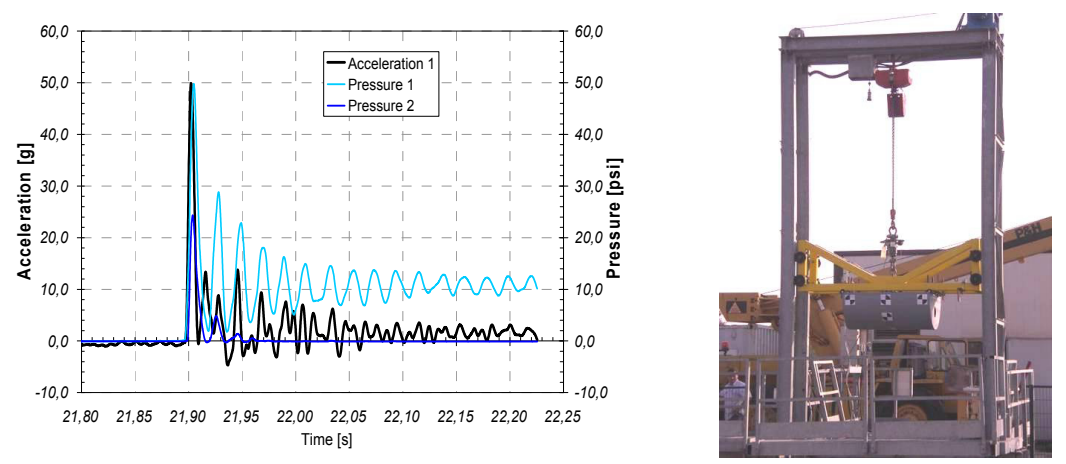

Figure 8: Impact on water of a deformable cylindrical shape $-V_{\text {impact }}=10 \mathrm{~m} / \mathrm{s}$ (CIRA's LISA test facility). 


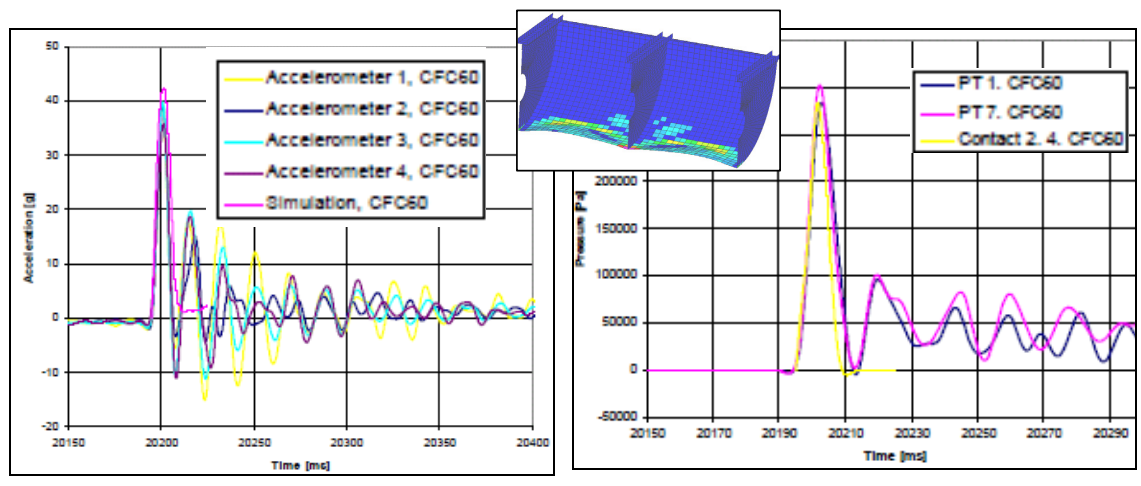

Figure 9: Numerical/experimental comparison for the $8 \mathrm{~m} / \mathrm{s}$ impact test.

The simulations for the 3 impact velocities lead to quite similar conclusions, hereafter summarized and illustrated in Figure 9 for the $8 \mathrm{~m} / \mathrm{s}$ impact velocity:

- The deformations of the test article are slightly over-predicted by the code (conservative analysis).

- Accelerations are conveniently predicted.

- $\quad$ Pressures are well predicted at soft areas i.e. where the test article deforms.

- Pressures are systematically over-predicted at stiff or slightly deformable areas (a refinement of the SPH network shall permit a better prediction).

\section{Conclusion}

These works performed within the GARTEUR Action Group AG15 aimed at evaluating the capacity of the SPH formulation to simulate fluid/structure interaction, with the objective to apply the method to the modelling of helicopter ditching [4]. In a preliminary step, one major goal of the group consisted in generating an experimental database including laboratory tests and tests on simple shapes, in order to collect relevant data for the validation of numerical models. Based on this experimental database, numerical works have established the relevance of the SPH formulation to represent the physics involved in fluid/structure interaction phenomenon and to predict the order of magnitude of most experimental observables (force, acceleration and pressure), however noting a tendency to some overestimation according to the considered data. Finally, SPH simulations also proved to be free of numerical instability and runnable over large run times, thus constituting one significant advantage compared to classical Finite-Element methods when modelling highly deformable media.

\section{Acknowledgements}

The authors wish to acknowledge the members of the GARTEUR Action Group AG15 "Improvement of SPH methods for application to helicopter ditching" for 
their support to the activities performed within the project. Authors also acknowledge the European Commission, the French Ministry of Defense and the Région Nord-Pas-de-Calais for their financial support to the present works.

\section{References}

[1] Langrand, B., Bayart, A-S., Chauveau, Y. \& Deletombe, E., Assessment of multi-physics FE methods for bird impact modelling - Application to a metallic riveted airframe, Int. Journal of Crashworthiness, 7(4), pp. 415-428, 2002.

[2] Groenenboom, P.H.L., Numerical Simulation of $2 D$ and 3D Hypervelocity Impact Using the SPH Option in PAM-SHOCK, Int. J. Impact Engng, Vol. 14, 1993, pp. 255-265.

[3] Anghileri, M., Castelletti, L., Francesconi, E., Pittofrati, M., Milanese, A., Rigid Body Water Impact: Experimental Tests and Numerical Simulations Using SPH Method, AHS2007.

[4] Toso-Pentecôte, N., Delsart, D., Vagnot, A., Assessment of the SPH method: Simulation of simple body shapes impacting on water and a PUMA helicopter ditching, ODAS Symposium - October 2009 - Berlin Germany. 